REVISTA DE LA ESCUELA DE CIENCIAS DE LA EdUCACIÓN, AÑo 15, NRO. 14, VOL. 2, JULIO A DICIEMRE DE 2020. PÁGINAS 131-142. ISSN 2362-3349 (EN LÍNEA). DECIDIR EL LENGUAJE. ESTRATEGIAS DE SUBJETIVACIÓN Y SUPERVIVENCIA COLECTIVA A TRAVÉS DE EXPERIENCIAS DE ARTE Y CULTURA EN CONTEXTOS DE ENCIERRO- CYNTHIA BUSTELO.

\title{
DECIDIR EL LENGUAJE \\ ESTRATEGIAS DE SUBJETIVACIÓN Y SUPERVIVENCIA COLECTIVA A TRAVÉS DE EXPERIENCIAS DE ARTE Y CULTURA EN CONTEXTOS DE ENCIERRO
}

\author{
Cynthia Bustelo* \\ Universidad de Buenos Aires, CONICET, Argentina \\ busteloc@gmail.com
}

Recibido: 16/08/2019 Aceptado: 28/12/2019

\section{Resumen}

Este trabajo presenta, describe y analiza la experiencia de formación y producción cultural llevada a cabo desde el Programa de Extensión en Cárceles (PEC), dependiente de la Secretaría de Extensión Universitaria y Bienestar Estudiantil (SEUBE) de la Facultad de Filosofía y Letras (FFyL) de la Universidad de Buenos Aires (UBA). Las estrategias y acciones llevadas adelante por el programa, están orientadas a construir herramientas y oportunidades para la inclusión social de las personas privadas de libertad y liberadas, y promover la defensa de los derechos humanos. Propongo poner a disposición las líneas de acción y reflexión que llevamos a cabo, recuperar los procesos de elaboración y puesta en marcha de las propuestas, para cruzar su devenir con algunas categorías construidas en mi tesis doctoral y otras elaboraciones conceptuales, políticas y epistemológicas que sustentan la tarea que llevamos a cabo. La reconstrucción de esta experiencia resulta un ejercicio formativo y tiene un valor en sí mismo, ya que permite dejar sistematizado algo de ese saber con el fin de dejar huella, para que otros equipos puedan conocer y, por qué no, retomar la experiencia que presentamos. Paralelamente, pondré a disposición cómo estas propuestas de apertura a la comunidad, permiten transformar no sólo la institución penitenciaria, sino la propia institución universitaria.

Palabras clave: experiencias de formación- contextos de encierro- prácticas socioculturales- extensión universitaria-lenguaje

\footnotetext{
*Doctora en Ciencias de la Educación por la Universidad de Buenos Aires. Licenciado en Ciencias de la educación (FFyLUBA). Becaria post-doctoral de CONICET. Es Coordinadora pedagógica y docente del Programa de Extensión en Cárceles (SEUBE-FFyL-UBA). Integrante de la Cátedra de Educación de Jóvenes y Adultos de la carrera de Ciencias de la Educación (FFyL-UBA) a cargo de la Dra. Lidia Rodriguez y la Dra. Esther Levy. Integra el Proyecto UBACyT: "Escribir en la cárcel: intervenciones con la literatura y otras formas de arte y organización", que dirige el Dr. Juan Pablo Parchuc. Codirige el UBANEX: "Prácticas y acciones socioeducativas y culturales en contextos de encierro: derechos e inclusión de personas privadas de libertad y liberadas". Forma parte del comité organizador del Encuentro Nacional de Escritura en la Cárcel. Fue coordinadora de distintos espacios educativos y culturales en contextos de encierro. Escribió y publicó artículos sobre la temática.
} 
REVISTA DE LA ESCUELA DE CIENCIAS DE LA EdUCACIÓN, AÑo 15, NRO. 14, VOL. 2, JULIO A DICIEMRE DE 2020. PÁGINAS 131-142. ISSN 2362-3349 (EN LÍNEA). DECIDIR EL LENGUAJE. ESTRATEGIAS DE SUBJETIVACIÓN Y SUPERVIVENCIA COLECTIVA A TRAVÉS DE EXPERIENCIAS DE ARTE Y CULTURA EN CONTEXTOS DE ENCIERRO- CYNTHIA BUSTELO.

\begin{abstract}
This work presents, describes and analyzes the experience of training and cultural production carried out by the Extension Program in Prisons (PEC), under the Secretariat of University Extension and Student Welfare (SEUBE) of the Faculty of Philosophy and Letters (FFyL ) of the University of Buenos Aires (UBA). The strategies and actions carried out by the program are aimed at building tools and opportunities for the social inclusion of persons deprived of liberty and freedom, and promoting the defense of human rights. I propose to make available the lines of action and reflection that we carry out, to recover the processes of elaboration and implementation of the proposals, to cross their future with some categories built in my doctoral thesis and other conceptual, political and epistemological elaborations that support The task we carry out. The reconstruction of this experience is a training exercise and has a value in itself, since it allows to systematize some of that knowledge in order to leave a mark, so that other teams can know and, why not, resume the experience we present. At the same time, I will make available how these proposals of opening up to the community, allow to transform not only the penitentiary institution, but the university institution itself.
\end{abstract}

Keywords: Formative experiences - Confinement contexts - Sociocultural practices - University extension - Language.

\title{
Prácticas y fundamentos de otros modos de construir Universidad
}

Este trabajo presenta, describe y analiza la experiencia de formación y producción cultural llevada a cabo desde el Programa de Extensión en Cárceles (PEC), dependiente de la Secretaría de Extensión Universitaria y Bienestar Estudiantil (SEUBE) de la Facultad de Filosofía y Letras (FFyL) de la Universidad de Buenos Aires (UBA). Pretendemos poner a disposición las líneas de acción y reflexión que llevamos a cabo, recuperar los procesos de elaboración y puesta en marcha de la experiencia universitaria desarrollada en contextos de encierro, para cruzar su devenir con algunas categorías construidas en la tesis doctoral y otras elaboraciones conceptuales, políticas y epistemológicas que sustentan la tarea que llevamos a cabo.

El Programa de Extensión en Cárceles (PEC) aborda problemas socioeducativos y culturales en contextos de encierro y vinculados con el sistema penal y la cárcel, a través de actividades orientadas a la defensa de los derechos humanos y la inclusión social de las personas privadas de libertad ambulatoria y liberadas. Este trabajo es realizado principalmente en centros universitarios y espacios educativos dentro de establecimientos penitenciarios federales y centros socioeducativos de régimen cerrado, aunque los alcances de la intervención abarcan otros espacios institucionales y políticos.

EI PEC se constituye formalmente en el año 2011. Depende de la Secretaría de Extensión Universitaria y Bienestar Estudiantil de la FFyL de la UBA y su fundación dio respuesta a la necesidad de reunir experiencias, saberes, acciones, que se venían llevando a cabo desde la facultad en los contextos de encierro. Desde aquel momento, se han realizado actividades en el Centro Universitario Devoto (CUD) alojado en el Complejo Penitenciario Federal de la Ciudad Autónoma de Buenos Aires y en el Centro Universitario Ezeiza (CUE) alojado en la localidad de Ezeiza, en el Complejo Penitenciario Federal I (CPFI) y el Complejo Penitenciario Federal IV de Mujeres (CPF IV), y en la Unidad 31-Centro Federal de Detención de Mujeres de Ezeiza; todos bajo la órbita del Servicio Penitenciario Federal (SPF). Tenemos presencia también en los tres centros socioeducativos de régimen cerrado (CSRC) actualmente activos en la CABA, dependientes del Consejo de los Derechos de Niños, Niñas y Adolescentes. Asimismo realizamos actividades extramuros, en la sede de Puan 480, el Centro de Innovación y Desarrollo para la Acción Comunitaria (CIDAC) y El Centro Cultural Paco Urondo para jóvenes que cumplen medidas penales morigeradas y/o personas que recuperaron su libertad.

Las actividades que llevamos a cabo constan de talleres, seminarios, y otras propuestas socioeducativas y de formación profesional, artística y cultural; actividades de investigación, extensión y docencia, en vínculo con cursos curriculares y extracurriculares; acciones institucionales, de vinculación social, articulación y difusión. De las actividades realizadas intramuros participan alrededor de cuatrocientas personas privadas de libertad ambulatoria y más de cuarenta estudiantes universitarios (en situación de encierro o no); aproximadamente setenta docentes, investigadores/as y graduados/as de distintas carreras de nuestra Facultad y otras unidades académicas, además de profesionales y activistas vinculados a la temática. Organizamos más de veinte talleres extracurriculares, cursos y espacios de orientación y formación en contextos de encierro (algunos repetidos en más de una locación). Realizamos también tareas de orientación y acompañamiento a estudiantes privados de libertad y liberados. Llevamos a cabo desde el 2016, una 
REVISTA DE LA ESCUELA DE CIENCIAS DE LA EDUCACIÓN, AÑo 15, NRO. 14, VOL. 2, JULIO A DICIEMRE DE 2020. PÁGINAS 131-142. ISSN 2362-3349 (EN LÍNEA). DECIDIR EL LENGUAJE. ESTRATEGIAS DE SUBJETIVACIÓN Y SUPERVIVENCIA COLECTIVA A TRAVÉS DE EXPERIENCIAS DE ARTE Y CULTURA EN CONTEXTOS DE ENCIERRO- CYNTHIA BUSTELO.

Diplomatura de pregrado en gestión sociocultural para el desarrollo comunitario ${ }^{1}$, primera oferta de estas características en contextos de encierro. A su vez, mantenemos o gestionamos nuevos convenios y vínculos de trabajo con otras unidades académicas, organismos estatales y organizaciones sociales. Participamos además de mesas de trabajo, congresos, y demás eventos científicos y de extensión universitaria; y organizamos encuentros, jornadas y espacios de debate y difusión intra y extramuros, de gran repercusión, como el Encuentro Nacional de Escritura en la Cárcel, que este año realizará su sexta edición.

Podemos enmarcar el programa en una larga tradición que entiende la extensión como el diálogo y la interacción de los conocimientos científicos con los saberes y necesidades de la comunidad o población de la que las universidades participan, abriendo espacios de compromiso y cooperación, con el objetivo de mejorar la calidad de vida de los grupos y personas que la integran. Si bien no desconocemos la advertencia que Paulo Freire y otra serie de autores y actores del campo popular que alimentaron la discusión, se dedicaron a explicitar sobre el término extensión, no será menester profundizar dicho debate en este trabajo. Según Juan Pablo Parchuc, Director del PEC y de las actividades de la FFyL en el Programa UBA XXII ${ }^{2}$ :

Las discusiones encaradas al respecto por las ciencias sociales y las llamadas humanidades están haciendo foco en la manera de expandir o tensar los límites (materiales, simbólicos, políticos) de los campos disciplinares y las prácticas académicas, desde una perspectiva crítica que a la vez cuestione los sentidos naturalizados dentro de sus propias instituciones y proponga modos de construcción y apropiación social de la cultura y el conocimiento para extender sus horizontes y posibilidades de intervención en el debate de ideas y la definición de las políticas educativas, sociales, económicas y culturales (Parchuc, 2015, p.18).

En este sentido, cabe la insistencia en destacar que nos alejamos de aquellas ideas o posiciones románticas y redentoras, que desde afuera se instalan para declamar cómo se deben hacer, realizar u organizar, en este caso, los espacios de encierro. Más bien se busca, "impulsar redes de co-formación, de sostén político-afectivo-intelectual, que promuevan la supervivencia colectiva como modo de resistencia, el despliegue de capacidades autogestivas y que movilicen y reivindiquen el saber de experiencia como saber pedagógico" (Bustelo, 2017, p.79).

La forma de trabajo que venimos llevando a cabo desde el PEC, y que está en franca consonancia con otras líneas de acción que (motorizadas por la extensión) integran la docencia y la investigación coordinadas y sostenidas por la SEUBE de la FFyL, es parte de una política universitaria que pretende, en conjunto con las organizaciones e instituciones barriales, comunitarias y experiencias autogestivas,

avanzar en procesos de innovación social, lo que supone una reconfiguración del proceso de gestión de conocimiento tradicional (tendencialmente auto-centrado y reproductivista) al generar una política de investigación en relación a problemas y demandas sociales cuya solución implica una abordaje interdisciplinario e intersectorial" (Trinchero y Petz, 2014, p.143).

Este escenario tensa los postulados académicos más elitistas y de corte positivista, rompe con tradiciones endogámicas enquistadas, interpela la institución universitaria, desafía a la comunidad educativa, democratiza el conocimiento, propone otro ángulo de mirada y construye otro modo de pensar y hacer universidad. A su vez, al habilitar y acompañar el tránsito por las experiencias universitarias para sectores que no se imaginaban este camino como una opción posible, genera resposicionamientos subjetivos que posibilitan la re-escritura de las propias historias singulares, y colectivas.

Si bien no existen cifras desagregadas sobre las características específicas de la población alojada en las cárceles, se toma como referencia los números a nivel nacional, según el último informe del Sistema Nacional de Estadísticas sobre la Ejecución de la Pena (SNEEP), publicado en 2017. Los datos muestran que hay alrededor de 85 mil personas detenidas en cárceles federales y provinciales de nuestro país. La población penal en Argentina creció de manera sostenida en las últimas dos décadas y tuvo un crecimiento pronunciado en los últimos tres años. Al momento de ser detenidos/as, la mayoría tenía historias o estaba viviendo en la pobreza y provenían de grupos marginalizados. El 83\% estaba desempleado o era trabajador de tiempo parcial. El $51 \%$ no tenía oficio ni profesión. En las cárceles, el $58 \%$ no tiene trabajo remunerado. El $76 \%$ no

\footnotetext{
${ }^{1}$ La Diplomatura supone un trayecto formativo anual, en el que puedan participar estudiantes universitarios y no universitarios, que otorga un diploma y les posibilita adquirir herramientas para sobrevivir en "el afuera" y fortalecer proyectos colectivos.

${ }^{2}$ EI programa UBA XXII depende de la Universidad de Buenos Aires, y dicta carreras de grado y actividades de Extensión en establecimientos del Servicio Penitenciario Federal desde el año 1985.
} 
REVISTA DE LA ESCUELA DE CIENCIAS DE LA EDUCACIÓN, AÑO 15, NRO. 14, VOL. 2, JULIO A DICIEMRE DE 2020. PÁGINAS 131-142. ISSN 2362-3349 (EN LÍNEA). DECIDIR EL LENGUAJE. ESTRATEGIAS DE SUBJETIVACIÓN Y SUPERVIVENCIA COLECTIVA A TRAVÉS DE EXPERIENCIAS DE ARTE Y CULTURA EN CONTEXTOS DE ENCIERRO- CYNTHIA BUSTELO.

participa de ningún programa de capacitación laboral y el $51 \%$ no participa de programas educativos. Sólo el $8 \%$ completó la escuela secundaria. Alrededor de un tercio $(29 \%)$ no terminó la escuela primaria. Un $3 \%$ estudia en la Universidad.

Más allá de los datos cuantitativos, es importante señalar que en el encierro las personas deben padecer diferentes problemáticas que dificultan su participación en las prácticas educativas: problemas burocráticos, escasez en la oferta educativa formal y no formal, limitaciones en las condiciones de infraestructura y recursos. También se encuentra restringido el acceso a las prácticas estéticas y culturales, tanto en términos de producción como de recepción. Por tanto, la educación resulta un derecho fundamental para las personas privadas de su libertad y liberadas, en tanto se constituye como puerta de acceso hacia otros derechos. Testimonio de ello es la importante convocatoria que tienen nuestras actividades, con gran cantidad de inscriptos por cuatrimestre (más de 1200 personas) y un alto nivel de impacto en términos de las discusiones y materiales que producen. Además, son un canal de ingreso al espacio universitario de estudiantes que todavía no concluyeron la escuela secundaria. En ese sentido, ante las restricciones en la inserción laboral de detenidos y liberados, los constantes obstáculos para acceder a la oferta educativa, las sistemáticas trabas con las que se encuentran para gozar de los otros derechos que no deberían suspenderse por estar privados/as de la libertad, resultan fundamentales las herramientas de formación, producción y acompañamiento que les permitan re-vincularse con sus estudios, fortalecer sus proyectos y modos de organización autogestivos.

Siguiendo este campo de problemas, cabe preguntarse qué tipo de experiencia universitaria estamos construyendo en esos contextos. Cómo contribuyen las distintas prácticas formativas socioculturales en la construcción de un cierto tipo de experiencia universitaria.

Según Jorge Larrosa, la experiencia tiene lugar cuando se transforma algo de lo que somos o sabemos:

Nuestra propia vida está llena de acontecimientos. Pero, al mismo tiempo, casi nada nos pasa. Los sucesos de actualidad, convertidos en noticias fragmentarias y aceleradamente caducas, no nos afectan en lo propio. Vemos el mundo pasar ante nuestros ojos y nosotros permanecemos exteriores, ajenos, impasibles. Consumimos libros y obras de arte, pero siempre como espectadores o tratando de conseguir un goce intrascendente e instantáneo. Sabemos muchas cosas, pero nosotros mismos no cambiamos con lo que sabemos. Esto sería una relación con el conocimiento que no es experiencia puesto que no se resuelve en la formación o la trans-formación de lo que somos (Larrosa, 2003, pp.28-29).

La experiencia universitaria que se construye en los distintos territorios pedagógicos en el (contra el y a pesar del) encierro, aloja experiencias de formación (Bustelo, 2017) inscriptas en la posibilidad de lucha y resistencia por la voz, por el derecho a pensar, por la necesidad de tomar decisiones en un contexto donde esto no es posible, por la identidad y la memoria que nos permite mayor conciencia histórica y sentido de futuro. Así, como enuncia Marcos Perearnau:

Los estudiantes que han transitado los grupos de talleres, quienes ya manejan la lengua universitaria y conocen tanto sus límites como sus alcances institucionales, se vuelven conscientes de que la transformación que se realizó por y a través de ellos, fue posible a partir de su participación en un proyecto común, educativo y cultural. El estudiante se reconoce a sí mismo en un relato colectivo, defiende los valores que comparte con una comunidad y es por lo tanto capaz de proyectarse. Es decir, ha logrado salir del estado de emergencia constante donde quedaba como resto y objeto, para comenzar a reconocerse como sujeto de derechos, capaz de reconocer a otros dentro del centro universitario, como fuera del mismo cuando recupera la libertad (Perearnau, 2017, p.10).

Construimos experiencias universitarias deseando que lo formativo sea el encuentro con las palabras, el diálogo de saberes, el intercambio con otros y otras. Donde lo biográfico cobre relevancia, donde incorporarse a la universidad signifique "salir de la cárcel", reponer derechos y entrar en diálogo con mundos desconocidos y desafiantes, pero que nos pertenecen. En esa clave construimos universidad en la cárcel a través de la experiencia sociocultural, comunitaria y colectiva, siguiendo la huella de Freire:

Educar y educarse en la práctica de la libertad, es tarea de aquellos que saben que poco saben -por esto saben que saben algo, y pueden así, llegar a saber más-, en diálogo con aquellos que, casi siempre, piensan que nada saben, para que éstos, transformando su pensar que nada saben en saber que poco saben, puedan igualmente saber más (Freire, 1993, p. 25). 
REVISTA DE LA ESCUELA DE CIENCIAS DE LA EdUCACIÓN, AÑo 15, NRO. 14, VOL. 2, JULIO A DICIEMRE DE 2020. PÁGINAS 131-142. ISSN 2362-3349 (EN LÍNEA). DECIDIR EL LENGUAJE. ESTRATEGIAS DE SUBJETIVACIÓN Y SUPERVIVENCIA COLECTIVA A TRAVÉS DE EXPERIENCIAS DE ARTE Y CULTURA EN CONTEXTOS DE ENCIERRO- CYNTHIA BUSTELO.

\section{"Adentro vos te acostumbras": de las marcas del encierro}

Propongo aquí describir las características y fundamentos de nuestra práctica a través de los espacios de formación y producción sociocultural que llevamos adelante desde el PEC, para luego profundizar en un cruce que vertebra nuestras conceptualizaciones: la experiencia de encierro y la experiencia de formación y producción cultural desde una perspectiva de resistencia, interrupción o resignificación de la situación de encierro punitivo (Bustelo 2017; Bustelo, Molina 2016; De Certeau 2007; Giroux 1992). Específicamente, me dedicaré a relatar algunas de las resonancias subjetivas y colectivas que emergen de aquello que sucede en ciertos dispositivos pedagógicos donde se dinamiza y convoca la palabra, poniendo en evidencia cómo se construyen estrategias de subjetivación, orientadas a la inclusión social de las personas privadas de su libertad, en los territorios pedagógicos en el (contra el y a pesar del) encierro. Así mismo, quedará en evidencia cómo estas instancias, impactan y fortalecen procesos de organización al interior de las cárceles, constituyéndose de este modo en estrategias de supervivencia colectiva.

Si bien se mencionaron más arriba algunas cifras que dan cuenta del contexto socio cultural característico de la mayoría de las personas privadas de la libertad (antes y durante su detención), resulta útil detenerme en una variable fundamental del contexto: las condiciones de vida en las cárceles. Las instituciones penitenciarias se caracterizan por una serie de fenómenos conflictivos y de vulneración de derechos: la violencia institucional, los fallecimientos por causas evitables, las condiciones insuficientes de alojamiento, que incluyen períodos prolongados en el encierro, condiciones deficientes de salubridad, higiene, alimento y calefacción, además del hacinamiento ${ }^{3}$. A estas problemáticas se suman las restricciones en el acceso y el ejercicio de derechos humanos básicos como la salud, la educación y el trabajo. Por otro lado, no solo las políticas de asistencia a los/as liberados/as y sus familias, a través de los patronatos y direcciones a cargo, son escasas y defectuosas sino que estas personas además cargan con el estigma de haber pasado por la cárcel, sufren las secuelas del encierro y tiene serias dificultades para continuar con sus estudios, conseguir empleo e incorporarse plenamente a la vida social en libertad. A esta estigmatización contribuye, en gran medida, un discurso público mediático y ligado a ciertos sectores conservadores, que identifica a estos grupos y personas como una amenaza para la ciudadanía, restringiendo su participación en la sociedad.

Los datos indicados hasta aquí muestran el campo de problemáticas sobre las que el PEC asienta su intervención. Puede especificarse también a través de los testimonios recuperados informalmente en clases por el equipo de trabajo y, más sistemáticamente, en los registros producidos en diversas instancias e investigaciones en curso, tanto como en la tesis doctoral en la que este trabajo se enmarca ${ }^{4}$.

Me interesa detenerme en cómo este campo de problemas supone efectos y deja marcas en las personas, para poder recuperar luego el intersticio, los puntos de fuga, que pueden acontecer a través de las experiencias de formación.

Irving Goffman realiza una caracterización sobre las Instituciones totales, en las que:

a) todos los aspectos de la vida se desarrollan en el mismo lugar y bajo la misma autoridad. b) todas las actividades se desarrollan junto con otros. c) todas las actividades están estrictamente programadas. d) todas las necesidades y todos los aspectos de la vida de los internos están sometidos a un plan predeterminado (Goffman, 2004, pp.21-22).

En ese sentido, intervenimos pedagógicamente en instituciones que se empeñan en:

Producir una reorganización total de la vida de las personas privadas de su libertad a partir de la destrucción y reconstrucción de su subjetividad. La regulación del tiempo, las condiciones de habitación, alimento, visita, comunicación, y medicalización, entre otras, responden a una lógica predeterminada con el objetivo de disciplinar y mantener el orden interno de las unidades. Las personas privadas de su libertad, dejan de tomar decisiones acerca de sus propias vidas: no está a su alcance decidir lo que comen, ni a qué hora, ni dónde duermen, ni con quién, ni de qué desean trabajar, ni en qué condiciones, ni lo que harán mañana, ni ninguna otra cosa (Bustelo, 2017, p. 61).

\footnotetext{
${ }^{3}$ CFR. Registros e informes de la Procuración Penitenciaria de la Nación, el Comité contra la Tortura de la Comisión Provincial por la Memoria y la Procuración de la Violencia Institucional del Ministerio Público.

${ }^{4}$ Las investigaciones a las que hago referencia están reunidas en los Proyectos UBACyT: "Escribir en la cárcel: teoría, marcos, acciones" (Programación Científica 2016), "Escribir en la cárcel: intervenciones con la literatura y otras formas de arte y organización" (Programación Científica 2018) y "Escribir en la cárcel: lenguas, políticas y comunidad", presentado recientemente para la Programación Científica 2020. Este trabajo se enmarca en mi tesis doctoral: "Experiencias de formación en contextos de encierro: un abordaje pedagógico desde la perspectiva narrativa y (auto) biográfica" Dirigida por los el Dr. Daniel Suárez y co-dirigida por el Dr. Juan Pablo Parchuc. Puede encontrarse en el Repositorio Digital de la FFyLUBA. http://repositorio.filo.uba.ar/handle/filodigital/4363
} 
REVISTA DE LA ESCUELA DE CIENCIAS DE LA EdUCACIÓN, AÑO 15, NRO. 14, VOL. 2, JULIO A DICIEMRE DE 2020. PÁGINAS 131-142. ISSN 2362-3349 (EN LÍNEA). DECIDIR EL LENGUAJE. ESTRATEGIAS DE SUBJETIVACIÓN Y SUPERVIVENCIA COLECTIVA A TRAVÉS DE EXPERIENCIAS DE ARTE Y CULTURA EN CONTEXTOS DE ENCIERRO- CYNTHIA BUSTELO.

Esto último, sumado al vínculo frágil e interrumpido con el mundo exterior, tiene para la vida adulta serias consecuencias. Rodolfo "Cacho" Rodríguez, sociólogo recibido en el CUD, pasó veintidós años preso, en distintos momentos de su vida. Su historia de formación, es una de las cinco que reconstruí para mi tesis doctoral. Su testimonio da cuenta, entre otras cosas, de los efectos que vengo relatando:

Todos coincidiríamos en que la vida en el encierro infantiliza, hace retroceder a las personas, despersonaliza, fragmenta la personalidad, te hace pelota. Pero además te genera una dependencia de la cual por ahí vos no sos consciente, pero mientras vos estás preso no pagás la luz, no pagás el gas, no pagás el teléfono, la comida mal que mal la tenés, el agua la tenés, el techo lo tenés. $Y$ cuando vos pusiste un pie en la calle, todo eso se te viene encima, eso hace que la gente reincida no importa si tenés un título de sociólogo, de jardinero, de plomero o de "tarjetas españolas" bajo el brazo (Bustelo, 2017, p. 116).

Liliana Cabrera, poeta e integrante de la organización YoNoFui que trabaja con mujeres privadas de su libertad y liberadas, construye un relato similar en torno a la salida, poniendo en evidencia cómo la cárcel entorpece la posibilidad de proyectarse y resolver conflictos adentro y afuera. Así, la libertad (paradójicamente) genera un estado de ansiedad y contradicción:

No me puse contenta te soy sincera, me agarró pánico, porque yo tenía que resolver ya algo que había pensado para seis meses después y que tampoco tenía idea de cómo iba a resolver porque la familia que no tenía no la iba a inventar en ese momento, trabajo... ¿de dónde saco?, ¿a dónde voy? porque adentro vos te acostumbras, a esa vida te acostumbras (Bustelo, 2017. p.100).

Es fundamental pensar la educación en contextos de encierro en esa clave: teniendo en cuenta que está inscripta en una maquinaria que infantiliza y ubica a la persona privada de su libertad en un lugar de tutela y minoridad, de dependencia, quitándole cotidianamente su poder de voluntad, de decisión sobre su cuerpo, sobre su tiempo, sobre sí mismo. Construir procesos de autonomía y responsabilización en un espacio donde la vida está sofocada de regulaciones (todo debe estar autorizado por el Servicio Penitenciario, jefes de módulos, celadores cercanos, o por los jueces que administran sus condenas, entre otros) y no hay lugar para la toma de decisiones, es una tarea prácticamente imposible. El encierro punitivo, que no contempla la privacidad ni el silencio; que no conoce de decisiones personales, proyectos de vida y voluntades cotidianas, apunta directamente sobre el mundo sensible y singular de cada persona.

Parte de este diagnóstico fundamenta también la necesidad de construir y reforzar canales y discursos alternativos para minimizar estos efectos, fortalecer estrategias de subjetivación que posibiliten armar otros vínculos allí para la generación de redes, para la producción de argumentos y palabras que permitan leer el mundo, decir el mundo, y participar en los debates de la agenda pública que diseña e implementa políticas penitenciarias. Es desde allí, que las actividades educativas, artísticas y culturales realizadas intramuros, que responden a la necesidad de dar a conocer y visibilizar las situaciones que se viven dentro de la cárcel y generar oportunidades para confrontarlas, creando alternativas más allá de la lógica punitiva del encierro, cobran especial relevancia.

Las siguientes páginas no desconocerán las arbitrariedades, las prácticas absurdas, abusivas y violentas, las sanciones, requisas, burocracias, efectos y marcas del encierro, las celdas de castigo, los traslados innecesarios, las prohibiciones, injusticias, la violencia institucional, las chicanas; lo que les sucede a las personas que allí adentro conviven, son maltratadas, infantilizadas, silenciadas y negadas en su autonomía y capacidad de decisión; aquellas personas que allí adentro esperan y desesperan. Hablará de todo ello, puesto en juego en el cruce con la experiencia de formación (Bustelo, 2017, pp.9-10). Narrar los fundamentos y resonancias de los talleres de formación y producción cultural en la cárcel, es contar otra historia posible.

\section{Hablar es decidir: tomá la voz}

Los dispositivos pedagógicos que articulan los saberes, las políticas y experiencias de formación y producción cultural vinculados a la lectura, la escritura y otros lenguajes artísticos se constituyen como estrategias de subjetivación individual tendientes a la inclusión social de las personas privadas de la libertad. Asimismo, interesa dejar en evidencia cómo estas experiencias inscriptas en territorios pedagógicos en el encierro (Bustelo 2017), fortalecen la organización para el desarrollo comunitario en cárceles (Bustelo y Parchuc, 2018).

Entre los años 2000 y 2015 aproximadamente, se constituyó un marco normativo y político de acción conjunta de la educación en contextos de encierro (Frejtman 2008), que implicó tanto el ingreso y la consolidación de las escuelas en las cárceles federales y provinciales, como el incremento de programas universitarios en contextos de encierro y la ampliación de la participación de las organizaciones sociales intramuros. Estas políticas y acciones contemplan no sólo la intervención pedagógica desde distintos ámbitos 
REVISTA DE LA ESCUELA DE CIENCIAS DE LA EDUCACIÓN, AÑo 15, NRO. 14, VOL. 2, JULIO A DICIEMRE DE 2020. PÁGINAS 131-142. ISSN 2362-3349 (EN LÍNEA). DECIDIR EL LENGUAJE. ESTRATEGIAS DE SUBJETIVACIÓN Y SUPERVIVENCIA COLECTIVA A TRAVÉS DE EXPERIENCIAS DE ARTE Y CULTURA EN CONTEXTOS DE ENCIERRO- CYNTHIA BUSTELO.

y posibilidades sino que incluyen el reconocimiento y la defensa de los derechos humanos y la inclusión social a través, por ejemplo, de la producción de publicaciones, talleres de oficios y armados de cooperativas en contextos de encierro. Esto permite evidenciar no solo el escenario de oportunidad normativo y político que se vino construyendo, sino la capacidad y la potencia de este tipo de actividades socio-educativas-culturales intramuros para atender a diversas problemáticas que atañen a la sociedad en su conjunto.

A los fines de este trabajo, quisiera detenerme en tres talleres extracurriculares específicos de la programación del PEC. Si bien todos se dictan en los Centros Universitarios Devoto y Ezeiza, se realizan para la población detenida en su conjunto, y no solo para aquellos que son universitarios. Como decía más arriba, esto hace parte de otros modos de construir experiencia universitaria, en clave colectiva, sociocultural, y en contextos de encierro.

El Taller de Narrativa se realiza desde el 2011. Existiendo desde los orígenes del programa, dio pie a su formación. El Taller Colectivo de Edición (TCE), que produce tres Revistas (La Resistencia en el Centro Universitario Devoto alojado en el Complejo Penitenciario Federal de la CABA; Los Monstruos Tienen Miedo en el Centro Universitario Ezeiza alojado en Complejo Penitenciario Federal I, Desatadas en el Centro Universitario Ezeiza alojado en el Complejo Penitenciario Federal IV). Por último, el Taller de Radio (cocoordinado por Radio La Tribu), que surgió de un proyecto de un grupo de estudiantes, en el marco de la Diplomatura de pregrado en Gestión sociocultural para el Desarrollo Comunitario, que desarrollamos desde el año 2016 en el CUD. El taller devino un programa de radio, denominado Radioculta, que sale al aire por la programación oficial de la radio 5

En los tres, se convoca a la palabra como herramienta de construcción, encuentro y (trans) formación. Se propone la toma de la palabra, y de decisión, como toma de posición. Para eso, se comparten herramientas, y se apela al estudiante privado de libertad como un sujeto de derechos, un sujeto de conocimiento, un sujeto de deseo, capaz de decidir qué quiere decir, cómo lo quiere decir, por quién le gustaría ser leído o escuchado. De esta manera, en los talleres de narrativa se los convoca tanto a leer como a producir textos. En el TCE se toman decisiones en vistas a conformar una política editorial (quiénes publican, cómo se construye el índice en cada número, qué tapa sale, qué retiraciones, con qué se ocupa la doble página central, entre otras). Para eso, se convoca a los estudiantes a construir entre todos y todas (docentes y participantes del taller), un colectivo editor, que sea responsable de tomar dichas decisiones, cada encuentro y cada cuatrimestre que la revista se publica. En el taller de radio, se coordina colectivamente cada programa que sale al aire, eligiendo temas, invitados/as, y organizando roles y dinámicas propias de la producción y difusión radial.

María José Rubín, docente y coordinadora del TCE, lo relata del siguiente modo:

EI TCE es, además del nombre de un curso extracurricular, un colectivo editor. Como tal, funciona de manera conjunta y las actividades desarrolladas en Ezeiza y en Devoto guardan una relación de parentesco entre sí: su conceptualización y sus propuestas de trabajo en el marco de ambos talleres surgen de reflexiones que ponen en común las problemáticas cotidianas de los dos espacios y que, a su vez, mantienen diálogo con la historia del TCE y su sentido original. Desde sus inicios, los equipos de coordinación (es decir, quienes ingresamos a los penales como docentes de la Facultad para desarrollar los cursos en los centros universitarios) han vivido ya varias generaciones de integrantes. Permanece, sin embargo, la vocación de llevar adelante una práctica horizontal, que permita a los compañeros inscritos en el curso tanto como a las coordinadoras (al momento de escritura de este texto, todas mujeres) participar en un pie de equidad de la formulación y confección de la revista, sin por ello dejar de atender a los límites de estas posibilidades. Estos límites están dados por diversos factores. Uno de ellos es, sin duda, "dónde estamos", la institución penitenciaria que nos enmarca y las muchas implicancias que esto conlleva (Rubin, 2019, p. s/f)

El pasaje de ser estudiante de un taller a transformarse en un colectivo editor, convoca la toma de decisiones, e interpela la responsabilidad de todas las personas que intervienen en aquello que se establece. A esto se suma la posibilidad de ser autor/a, ser publicado/a en la revista, o "salir al aire". Esta instancia les permite ubicarse en la escena desde su propia voz, como personas posicionadas sobre una temática, que los/las convoca y los/las invita a "decir algo". Como afirma Rubin:

\footnotetext{
${ }^{5}$ FM La Tribu es un colectivo de comunicación alternativa. El programa Radioculta se emite los días Jueves a las 23.59 hs. Cada episodio se sube a https://www.facebook.com/radioculta/ y Spotify, entre otros soportes.
} 
REVISTA DE LA ESCUELA DE CIENCIAS DE LA EdUCACIÓN, AÑo 15, NRO. 14, VOL. 2, JULIO A DICIEMRE DE 2020. PÁGINAS 131-142. ISSN 2362-3349 (EN LÍNEA). DECIDIR EL LENGUAJE. ESTRATEGIAS DE SUBJETIVACIÓN Y SUPERVIVENCIA COLECTIVA A TRAVÉS DE EXPERIENCIAS DE ARTE Y CULTURA EN CONTEXTOS DE ENCIERRO- CYNTHIA BUSTELO.

Una de nuestras herramientas principales para convocar nuevos integrantes es la propia revista impresa. Los itinerarios que describe, de mano en mano, al interior de la institución carcelaria, permiten que muchos lectores se interesen en la posibilidad de convertirse en participantes activos. Las revistas impresas nos permiten incluso trascender las paredes de los penales en los que desarrollamos las instancias del Taller y recibir colaboraciones de compañeros y compañeras que se encuentran en otros penales o que están en libertad (Rubin, 2019, s/p).

En palabras de Lucas Adur, Luciana De Mello y María Elvira Woinilowicz, docentes del Taller de Narrativa, también acontece un movimiento que transita entre lo singular y lo colectivo:

Podríamos pensar cualquier instancia de lectura como un acto íntimo de comunicación, un encuentro que cruza al lector con el cuerpo del relato, con el autor y su contexto, donde no solo se pone en juego el cúmulo de lecturas en tanto textos leídos, sino que también está ahí la propia experiencia de lectura del mundo como una herramienta fundamental, en permanente uso y expansión. En principio, la idea que le dio origen al Taller de Narrativa del Centro Universitario Devoto (CUD) fue hacer colectiva esta actividad íntima (Adur, De Mello y Woinilowicz, 2016, p.111)

A estos testimonios de docentes, podemos sumar un conjunto de trabajos que se preguntan por los efectos simbólicos de la lectura, la escritura y otras prácticas y experiencias artísticas, educativas y culturales, sobre los sujetos y las instituciones que los mantienen encerrados: cómo actúan produciendo reposicionamientos subjetivos, trastocando escenas y situaciones, a partir del trabajo con la literatura y otros lenguajes, técnicas o formas de organización (Bustelo 2017; Petit, 2003; Perearnau 2017;Trucco y Pansera 2016). Podríamos partir de las discusiones sobre la construcción del "delincuente" y su productividad social (Foucault 1991; Garland 2005; Goffman 2004; Wacquant 2004; Zaffaroni 2006). O su problematización y contrapunto cuando quienes son interpelados por estas representaciones logran realizar otras construcciones de sí mismos (Butler 2009, 2004, 1993) o, imaginar (inscriptos en otras condiciones de producción del discurso y la escucha) "mundos de transformación colectiva" (Delfino y Parchuc 2017, p.109). En esta línea, podríamos preguntarnos qué tipo de dispositivos configuran relaciones y procesos de exclusión y cuáles, al propiciar herramientas y construir otros marcos de acción para la producción y circulación de los saberes, dan margen (Parchuc, 2014b), abren posibilidades y crean nuevos horizontes y oportunidades de inclusión, para leer y decir el mundo, para leer el propio mundo, para decir la palabra propia (Freire, 2010). Asimismo, cabe destacar cómo estos dispositivos pedagógicos en cárceles, facilitan en muchos casos la continuidad o terminalidad de los trayectos educativos escolares. Esta población suele estar signada por trayectorias escolares interrumpidas, fragmentadas, dañadas. Los dispositivos pedagógicos permiten a su vez, no sólo facilitar el acceso y continuidad de los recorridos escolares, sino incorporar una serie de prácticas, discursos y saberes de la vida académica, que pueden ser puerta de ingreso a una carrera universitaria intramuros, y posibilidad de continuidad una vez obtenidas la libertad.

Siguiendo esta línea argumental, las experiencias socioculturales en la cárcel y en espacios de vulneración social, muchas veces crean oportunidades para la recuperación de la palabra, habilitando otras identidades o versiones de la propia historia, a contrapelo de las que prevalecen en medios hegemónicos sentencia e informes criminológicos (Frejtman y Herrera, 2010). Ese movimiento no trata de eludir o negar la responsabilidad (Segato 2003), sino que abre un espacio para volver a narrar la (auto)biografía en un sentido distinto al supuesto destino fijado por la condena; posibilitando la (re)escritura de la historia individual y colectiva. Como mencioné más arriba, se habilita también un reposicionamiento subjetivo, en tanto la persona privada de su libertad pasa a ser un/a estudiante, y desde ese lugar, un/a autor/a. Así, se asiste a un movimiento, un tránsito, un pasaje que tiene implicancias tanto singulares como colectivas, ya que permiten dinamizar los vínculos que se construyen y sostienen intramuros tanto como desdibujar las dinámicas y roles propios del contexto. Esto impacta de lleno en los procesos de organización colectiva para ejercer sus derechos y mejorar sus condiciones de vida. En definitiva, se reivindica las posibilidades que abren las experiencias socioculturales para desmontar estigmas y producir herramientas que abran horizontes, permitan imaginar otros mundos posibles y exploren formas de pensar la vida en común y construir comunidad (Williams, 2001, pp. 262-275). Compartimos con Parchuc que:

Sin duda, la palabra tiene un valor diferencial dentro de la cárcel. Llena expedientes, legajos y oficios. Atraviesa los muros en forma de correspondencia, se graba en las paredes, circula como rumor (la bemba), pasa de mano en mano, vuela o se descuelga de una ventana a otra como paloma. Define sanciones y condenas y puede usarse para insultar y verduguear. Pero también es una herramienta creativa y de resistencias, e incluso puede llegar a ser un medio de inclusión. La escritura en la 
REVISTA DE LA ESCUELA DE CIENCIAS DE LA EdUCACIÓN, AÑo 15, NRO. 14, VOL. 2, JULIO A DICIEMRE DE 2020. PÁGINAS 131-142. ISSN 2362-3349 (EN LÍNEA). DECIDIR EL LENGUAJE. ESTRATEGIAS DE SUBJETIVACIÓN Y SUPERVIVENCIA COLECTIVA A TRAVÉS DE EXPERIENCIAS DE ARTE Y CULTURA EN CONTEXTOS DE ENCIERRO- CYNTHIA BUSTELO.

cárcel es una "bocanada" (Domenech y Nazar Ondas de Hiroshima: 5) o al menos un "cacho de libertad" (María Medrano Yo no fui: 9) (Parchuc, 2014, p.75).

Los y las propias estudiantes privadas de la libertad dejan ver en sus escritos esta misma constelación de ideas que Parchuc reúne y conceptualiza. Liliana Cabrera también afirma que la escritura le posibilitó procesos subjetivos que tuvo efectos en su cuerpo, en su lenguaje, y en su forma de ver la vida. Ella cuenta cómo logró tramitar su tartamudeo, rearmar su identidad y construir comunidad, a través de la escritura:

La primera vez que fui había un grupo que escribía bárbaro. Yo era muy tímida, tartamudeaba y no había escrito nada de poesía. Habían dado un disparador, yo escuché y había que traerlo para la próxima semana. Había que escribir sobre un ambiente, sobre un lugar. Y escribí un poema que ahora figura en Obligado Tic Tac ${ }^{6}$, yo escribí sobre el pabellón comunitario, el de ingreso, el 17 . Y cuando las compañeras lo comentaban, no es que había mala onda, era la dinámica del taller, pero uno no está acostumbrado, y me hacían sugerencias y yo pensaba que nos les había gustado. O por ejemplo, no quería leer yo y se los daba a una compañera para que las lea, porque me daba vergüenza el tartamudeo. Hasta que a medida que fue pasando el tiempo me di cuenta que no pasaba nada, que ya las conocía. Y de hecho empecé a no tartamudear tanto. La escritura entre otras cosas me ayudó a eso (Bustelo, 2017, pp. 87-88).

En un taller de literatura de uno de los CSRC de la CABA también organizado en el marco del PEC, se construyó un poema colectivo que proponía la siguiente pregunta ¿Por qué escribimos?:

Porque con la literatura puedo inventar otra realidad.

Porque aprendo más cosas: aprendo a leer, aprendo a escribir.

Porque me relajo.

Porque el día de mañana le puedo enseñar a mis hijos.

Porque tenemos muchas preguntas para hacernos. colectivo:

Con idéntica consigna, se construyó en el CUD en el marco del taller de narrativa, el siguiente poema

Por qué escribimos

si quizás nadie lo lea

o quizás nadie lo escuche.

Por qué escribimos si nuestra profesión

capaz no sea ser escritores.

¿Escribimos porque resulta más fácil que decirlo?

¿Porque tenemos alma de poeta?

¿Porque nos gusta escribir?

Porque la memoria y el futuro

Porque hay tiempo y no

Porque estamos juntos

Porque estamos solos

Para que algo quede.

Escribimos para que los recuerdos no se pierdan en la memoria,

para que no se escondan en el tiempo

para que vivan en la historia

Escribimos porque es barato

y seguimos escribiendo por obstinación,

por no dejar de lado para siempre los mundos soñados.

Escribimos para que la vida duela menos.

Escribimos porque estamos enfermxs de soledad, por egoísmo.

Escribimos para establecer un vínculo al futuro

Para sentir que podemos decir algo, que tenemos algo para decir

Algo bello, algo fatal, algo extraordinario

Para pensar que no existe la muerte

Escribimos porque duele menos que clavarse la birome en la piel

${ }^{6}$ Liliana Cabrera escribió tres libros mientras estaba detenida en la U.31: Obligado Tic Tac, Bancame y Punto y Tu nombre escrito en tinta china. 
REVISTA DE LA ESCUELA DE CIENCIAS DE LA EdUCACIÓN, AÑo 15, NRO. 14, VOL. 2, JULIO A DICIEMRE DE 2020. PÁGINAS 131-142. ISSN 2362-3349 (EN LÍNEA). DECIDIR EL LENGUAJE. ESTRATEGIAS DE SUBJETIVACIÓN Y SUPERVIVENCIA COLECTIVA A TRAVÉS DE EXPERIENCIAS DE ARTE Y CULTURA EN CONTEXTOS DE ENCIERRO- CYNTHIA BUSTELO.

Escribimos porque nada bueno se puede hacer con la tristeza, salvo, quizás, escribir poemas ${ }^{7}$.

En este sentido sugiero pensar los talleres de formación y producción cultural que dan lugar a experiencias de formación, como experiencias vitales. En estos espacios se proponen otras lógicas, donde la escritura, la lectura, la producción y difusión son entendidas en términos de reposicionamientos subjetivos que fortalecen procesos de organización colectiva. Donde la práctica formativa se funde y sustenta en el encuentro, en la construcción de un nosotros que co-forma un espacio. Donde el diálogo de saberes se constituye como parte fundamental. Donde la decisión del otro y del colectivo importa, tiene un peso, arma trama y construye relato de un territorio.

Podemos escribir para nosotros, para no olvidar, para no tartamudear, para hacernos preguntas, 0 simplemente para relajarnos. Pero también podemos escribir en colectivo, para publicar y visibilizar, para fortalecer(nos), aprender (nos), conocer(nos) y reconocer(nos). Podemos escribir y hablar en un micrófono para que nos escuchen nuestros seres queridos, o para que nos escuche gente que no imaginamos. Para generar empatía o para escandalizar. Para ser protagonistas, o héroes colectivos. Podemos escribir, publicar, hablar, en soledad. O podemos hacerlo en colectivo, para construir un relato en y sobre el territorio, o simplemente para que la cárcel, duela menos. Esa es la propuesta y sustento epistemo-político de los talleres de formación y producción cultural que llevamos adelante desde el PEC.

\section{Palabras finales: la imaginación no se negocia}

En este trabajo me propuse describir y analizar la propuesta formativa del PEC en tanto dispositivo pedagógico de formación y producción cultural. En ese sentido, puse a disposición el marco teórico, político y pedagógico desde el que pensamos la construcción de esta experiencia de extensión universitaria tan peculiar. Y los fundamentos, prácticas y resonancias de los talleres pensados como experiencias de formación donde se habilitan procesos de construcción de conocimiento de sí, y conocimiento colectivo. En ese sentido, podemos afirmar que los talleres son espacios epistémicos, con efectos resonantes, que dejan huella sobre todas las personas que allí participamos. Que incide sobre nuestro sistema de creencia sobre lo que somos, sabemos y hacemos. Como señalé en la tesis doctoral:

Es posible indicar que los Centros Universitarios Devoto y Ezeiza se constituyen como territorios pedagógicos que propician no sólo experiencias de aprendizaje y prácticas universitarias, tendientes a construir "marcas universitarias" y "subjetividad de estudiantes" sino experiencias de formación intelectual, reflexiva, política, artística y colectiva que atentan e intentan suspender momentáneamente, o bien, poner entre dicho, el encierro punitivo. El CUD y el CUE son además de centros de formación, usinas de producción artística y cultural (Bustelo, 2017, p. 211).

Tanto las revistas, los textos y libros publicados ${ }^{8}$, como el programa de radio, producen hechos socio-culturales, arman redes políticas-afectivas y construyen puentes integradores (adentro/afuera, ahora/después). Así, desde los talleres que los contienen, se anclan y hacen de sus espacios territorios pedagógicos en el (contra él y a pesar del) encierro (Bustelo, 2017). A su vez, sostienen la construcción de otro tipo de experiencias universitarias, ligadas a la formación y la producción sociocultural donde lo formativo es lo común, el encuentro, lo que allí sucede entre quienes co-forman el espacio. Donde se reivindica la pregunta, el aprendizaje colectivo, la problematización acerca del saber y de las prácticas académicas. Y para ello, para construir experiencias vitales, que disloquen certezas, que conmuevan biografías y permitan reescribirlas, se necesita decidir el lenguaje. El lenguaje permite interrogarnos y poner en cuestión la realidad. Como afirmaba Liliana Bodoc en una conferencia denominada "La literatura en los tiempos del oprobio"'.

\footnotetext{
${ }^{7}$ Esta es una versión abreviada del poema, que fue grabado por Liliana Herrero para el IV Encuentro Nacional de Escritura en la Cárcel: https://www.facebook.com/escrituraenlacarcel/videos/1996996167252313/ (última consulta: 15/8/19)

${ }^{8}$ Las Revistas que mencionamos son las producidas por el TCE. Los libros publicados: Ninguna Calle Termina en la Esquina, surgido del taller de narrativa del CUD. Expresos Literarios: antología de jóvenes escritores en contextos de encierro, surgido del taller de literatura en el CSRC Manuel Belgrano y los libros publicados por WK, a través de la editorial Tren en Movimiento y la colaboración de la FFyL-UBA: 79, el ladrón que escribe poesías, 118, y 48 el muerto que escribe cuentos.

${ }^{9}$ La conferencia: "La literatura en tiempos del oprobio" dictada por Liliana Bodoc, fue Conferencia de apertura de las XVII Jornadas "La literatura y la escuela" de Jitanjáfora ONG. Mar del Plata, 7 de abril de 2017. Puede encontrarse en: https://www.youtube.com/watch?v=qsZ1S1iSBSA (última consulta: 15/8/19)
} 
REVISTA DE LA ESCUELA DE CIENCIAS DE LA EDUCACIÓN, AÑo 15, NRO. 14, VOL. 2, JULIO A DICIEMRE DE 2020. PÁGINAS 131-142. ISSN 2362-3349 (EN LÍNEA). DECIDIR EL LENGUAJE. ESTRATEGIAS DE SUBJETIVACIÓN Y SUPERVIVENCIA COLECTIVA A TRAVÉS DE EXPERIENCIAS DE ARTE Y CULTURA EN CONTEXTOS DE ENCIERRO- CYNTHIA BUSTELO.

Hablar es decidir, es imposible que no haya decisión en el acto de hablar. Si nosotros no decidimos, va a decidir el pensamiento hegemónico. Si como personas desestimamos la importancia de decidir nuestra palabra va a hablar por nosotros el pensamiento hegemónico y entonces vamos a decir: "algo habrán hecho", "quién la mandó a usar minifalda", "los maestros tienen tres meses de vacaciones", "no son treintamil". Decidir el lenguaje es decidir lo que somos y lo que hacemos. En estos tiempos donde hay un ataque casi de ficción contra la educación plural y sensible, contra la gracia y la ternura. Nos toca decidir el lenguaje y ejercerlo con el mayor coraje (Bodoc, 2017, s/p).

Estela Quintar lo define como:

La posibilidad de promover sujetos autónomos, potentes, capaces de extender las alas de la imaginación para saber que sí es posible cambiar las propias circunstancias y las de la comunidad en la que se desarrolla, es una opción de trabajo constante. Es, de alguna manera, asumir la conciencia histórica como derecho y deber. (...) El gran desafío es, justamente, la lucha por el significado, por nombrar lo no nombrado y generar permanentemente alternativas que nos permitan no negociar ni la mirada ni la voz ni la imaginación radical. (Salcedo, 2009, p.124)

Si estos dispositivos pedagógicos permiten decidir, al menos, el lenguaje: ¿Qué le pasa al sujeto con esa decisión? ¿Y con la decisión colectiva? ¿Qué puede movilizar, mover, desarmar, corromper, conocer, estructurar, aprender, desear, transformar en ese proceso de formación y producción? ¿Qué aprendió en el taller? Compartir y construir en colectivo instala una dinámica grupal, circular, en la que el otro no es enemigo y mi forma de mirarlo, pensarlo, nombrarlo y compartir con él, resonará en mi forma de mirar la humanidad y vincularme con ella, y con el mundo. El mundo no es así una cosa externa, los otros y las otras no son una amenaza, los saberes no enajenan y cosifican, sino que arman trama y comunidad. Y en eso, la palabra política, la palabra pedagógica, la palabra decidida, tiene un rol fundamental.

Decidir el lenguaje es tomar la palabra, para leer el mundo y poder decir el mundo con la palabra propia, con la palabra que comunica, que abre, la que está ahí (silenciosa o verborrágica) y la que emerge del encuentro. Decidir el lenguaje no sólo para decidir algo en un contexto donde no se puede decidir nada, sino para decidir lo primordial, lo que nos constituye como seres humanos, lo que nos permite relatarnos. Decidir el lenguaje para poder contarle a las familias de qué se trata esa nueva experiencia que están transitando con la universidad, para poder defenderse ante el juez, para poder narrar lo que aprendieron e invitar a otros compañeros al CUD y al CUE, para no sentirse tan solos, tan solas, para encontrarse en esa palabra propia y colectiva. Decidir el lenguaje para que no digan otros por nosotros/as, para describir ese espacio que no es la cárcel, pero tampoco es puramente la universidad, para transitar sus intersticio y poder nombrarlo. Para escribir desde el primer día, escribir como opción, escribir para no olvidar, y en definitiva así, transformar espacios, identidades y re-escribir historias. La propia y la colectiva.

\section{Referencias bibliográficas}

Adur, L.; De Mello, L. y Woinilowicz, M. E. (2016). Narrar es como jugar al póker. Reflexiones sobre autoría, lectura, ficción y valor literario desde el Taller de Narrativa de Devoto. En: Espacios de Crítica y Producción, núm. 52.

Bustelo, C. y Molina, L. (2016). La experiencia del taller de educación popular como territorio político-pedagógico. En: Revista Espacios. №52.

Bustelo, C. (2017). Experiencias de formación en contextos de encierro: un abordaje pedagógico desde la perspectiva narrativa y (auto)biográfica. En: Repositorio de la Facultad de Filosofía y Letras-UBA. http://repositorio.filo.uba.ar/handle/filodigital/4363 (consulta: 25 de Julio de 2019)

Bustelo, C. y Parchuc, J.P. (2018). Estirar la reja: libro, voces y proyecciones”. En Saberes en diálogo. Experiencias de formación y gestión sociocultural en la cárcel. Ciudad Autónoma de Buenos Aires: EPISEC.

De Certeau, M. (2007). La invención de lo cotidiano. México: Universidad lberoamericana.

Delfino, S. y Parchuc, J.P. (2017). Narrar para re escribir: experiencias pedagógicas en contextos de encierro. En: Gerbaudo et Tosti (eds.) Nano-Intervenciones con la literatura y otras formas del arte, pp. 109-142. Santa Fe: Universidad Nacional del Litoral.

Dirección Nacional de Política Criminal en materia de Justicia y Legislación Penal. Subsecretaría de Política Criminal Secretaría de Justicia. Ministerio de Justicia y Derechos Humanos (2018). "Sistema Nacional de Estadísticas sobre ejecución de la pena. Informe anual 2017". Recuperado de: https://www.argentina.gob.ar/sites/default/files/informe_sneep_spf_2017.pdf. (Consulta: 24 de Junio de 2019).

Foucault, M. (1991). Las redes del poder. Buenos Aires: Almagesto.

Freire, P. (1993). ¿Extensión o comunicación? La concientización en el medio rural. México: Siglo XXI.

Freire, P. (2010). Pedagogía del Oprimido. Buenos Aires: Siglo XXI.

Frejtman, V. y Herrera, P. (2010). Pensar la educación en contextos de encierro. Aproximaciones a un campo de tensión. Mod. 1. Buenos Aires: Ministerio de Educación de la Nación. 
REVISTA DE LA ESCUELA DE CIENCIAS DE LA EdUCACIÓN, AÑo 15, NRO. 14, VOL. 2, JULIO A DICIEMRE DE 2020. PÁGINAS 131-142. ISSN 2362-3349 (EN LÍNEA). DECIDIR EL LENGUAJE. ESTRATEGIAS DE SUBJETIVACIÓN Y SUPERVIVENCIA COLECTIVA A TRAVÉS DE EXPERIENCIAS DE ARTE Y CULTURA EN CONTEXTOS DE ENCIERRO- CYNTHIA BUSTELO.

Garland, D. (2005). La cultura del control. Crimen y orden social en la sociedad contemporánea. Barcelona: Gedisa. Giroux, H. (1992). Teoría y resistencia en educación. México: Siglo Veintiuno.

Goffman, E. (2004). Internados: ensayos sobre la situación situación social de los enfermos mentales. Buenos Aires: Amorrortu.

Parchuc, J.P. (2014a). Escribir en la cárcel: acciones, marcos, políticas. En: Boletín de la Biblioteca del Congreso de la Nación, 128, pp.67-81.

Parchuc, J.P. (2014b). Dar margen: teoría literaria, crítica e instituciones. Dar Margen: teoría literaria, crítica e instituciones. En El taco en la Brea Revista anual del Centro de Investigaciones

Parchuc, J. P. (2015). La Universidad en la cárcel: teoría, debates, acciones. Redes de Extensión, pp.18-36. №1.

Parchuc et al. (2016). 30 aniversario del Programa UBAXXII. En: Espacios de Crítica y Producción, núm. 52. En línea: http://revistascientificas.filo.uba.ar/index.php/espacios. Consulta; 24 de Junio de 2019

Perearnau, M. (2017). Agrandaré mis prisiones. De la causa penal a una causa universitaria, cultural y colectiva. Una elaboración de la experiencia universitaria del CUSAM a partir de las estrategias de subjetivación de los talleres artísticos. En: M. Chiponi; M. Manchado, y R. Castillo (eds.) A pesar del encierro. Prácticas políticas, culturales y educativas en prisión. Rosario: Espacio Santafesino + Industrias Creativas, Ministerio de Innovación y Cultura de Santa Fe.

Petit, M. (2003). "La lectura íntima y compartida", I Jornadas Aragonesas de Bibliotecas Escolares y Promoción de la Lectura. 10ำ Aniversario de «Leer juntos», Ballobar, 2003.

Rubin, M.J. (2019). Políticas y dinámicas editoriales en contextos de encierro: la experiencia del Taller Colectivo de Edición. En: Escribir en la cárcel: Escribir en la cárcel. Prácticas y experiencias de lectura y escritura en contextos de encierro. Ciudad Autónoma de Buenos Aires, Facultad de Filosofía y Letras, MIMEO.

Trinchero, H., Petz, I. (2014). La cuestión de la territorialización en las dinámicas de integración UniversidadSociedad. Aportes para un debate sobre el "academicismo". En Centro de Estudios Interdisciplinarios en Etnolingüística y Antropología Socio-Cultural. En Papeles de Trabajo № 27.

Salcedo, J. (2009). Pedagogía de la potencia y didáctica no parametral. Entrevista con Estela Quintar. En Revista Interamericana de Educación de Adultos, vol. 31, núm. 1, pp. 119-133. Centro de Cooperación Regional para la Educación de Adultos en América Latina y el Caribe. Pátzcuaro, México

Segato, R. (2003). El sistema penal como pedagogía de la irresponsabilidad y el proyecto "habla preso: el derecho humano a la palabra en la cárcel". Brasilia: Departamento de Antropología, Universidad de Brasilia, 2003. En línea: http://lanic.utexas.edu/project/etext/llilas/cpa/spring03/culturaypaz/segato.pdf (Consulta: 24 de Junio de 2019)

Trucco, M. J. y Pansera, C. (2016). Los móviles. Modus operandi de acciones dramáticas en cárceles. Buenos Aires: Edición de Artes Escénicas.

Wacquant, L. (2004). Las cárceles de la miseria. Buenos Aires: Manantial.

Williams, R. (2001). Cultura y sociedad. Buenos Aires: Nueva Visión.

Zaffaroni, E. R. (2006). El enemigo en el derecho penal. Buenos Aires: Ediar. 